\title{
A COMPARISON OF PRODUCTION PLANNING FORMULATIONS WITH EXOGENOUS CYCLE TIME ESTIMATES USING A LARGE-SCALE WAFER FAB MODEL
}

\author{
N. Baris Kacar \\ Operations Research Consulting Group \\ SAS Institute \\ Cary, NC 27513, USA
}

\author{
Lars Mönch \\ Department of Mathematics and Computer Science \\ University of Hagen \\ 58097 Hagen, GERMANY
}

\author{
Reha Uzsoy \\ Edward P.Fitts Department of Industrial and Systems Engineering \\ North Carolina State University \\ Raleigh, NC 276-7906, USA
}

\begin{abstract}
A key parameter of the linear programming (LP) models widely used for production planning in industry and academia are the lead times, the estimated delay between material becoming available to a resource and the completion of its processing at that resource. Lead times are commonly treated as exogenous, workload-independent parameters and assumed to be integer multiples of the planning period. Although formulations with non-integer lead times have been proposed in the past, we are not aware of any studies that systematically evaluate the benefits of using non-integer lead times in LP models. In this paper we implement LP models to plan the releases of wafers into a large-scale wafer fabrication facility and compare the performance of LP models with and without non-integer lead times by simulating the execution of the resulting release plans. We find that the models with non-integer lead times yield substantially improved performance, and are quite straightforward to implement.
\end{abstract}

\section{INTRODUCTION}

Linear programming (LP) models have been widely used since the late 1950s to address the problem of production planning. Production planning involves allocating the capacity of production resources to competing products, or operations of products, over time in order to optimize some measure of factory performance. The key decisions are the amount of raw material to release into the production system over time such that the output of the system can be used to meet demand in the most advantageous manner. Extensive descriptions of models of this type are given by Johnson and Montgomery (1974), Hackman (Hackman 2008), Missbauer and Uzsoy (2010) and Voss and Woodruff (2003), among others.

A key issue in production planning models of this type arises from the fact that the flow of material through capacity-constrained production facilities involves substantial delays that are mostly due to queueing for congested resources. In semiconductor wafer fabrication, the application motivating this study, the average cycle time of the overall process can be of the order of several weeks, requiring that models take these delays explicitly into account. The estimates of cycle times used in production planning models will be referred to as lead times.

Queueing models of manufacturing systems (Buzacott and Shanthikumar 1993; Hopp and Spearman 2008) have shown that the average cycle time, the time between a unit of work arriving at a resource and 
its completing its processing there, increases nonlinearly with resource utilization. Thus the cycle time at a given production resource at any point in time is a complex function of the capabilities of the equipment, such as processing batch size, processing time and setup time, as well as the stochastic processes governing the arrival and service times at the resources and the decision rules used to allocate resources among competing jobs in the queue. The amount of time elapsing between a unit of work being released into the production system and its arriving at a specific resource is thus a random variable whose distribution depends on the history of all work releases into the system until that point in time as well as many other sources of uncertainty in the production process. The lead times used in production planning models are thus estimates of some appropriately selected statistic of this distribution. The fundamental underlying difficulty is that the lead time is determined by, and not an input into, the planning process, since the amount of work released into the system over time determines resource utilization and hence cycle times.

Although ongoing research is addressing the problem of planning with workload-dependent lead times (Pahl et al. 2005; Asmundsson et al. 2006; Pahl et al. 2007; Asmundsson et al. 2009; Missbauer and Uzsoy 2010), many planning systems in current operation, including the widely used Material Requirements Planning (MRP) procedure (Orlicky 1975; Baker 1993), require deterministic lead time estimates as parameters. However, the estimation of lead times for use in production planning models is often not straightforward. Simple thought experiments, borne out by observation of industrial systems and simulation models, should convince the reader that choice of lead times can affect the cost of operating the production system. Underestimating lead times will result in releasing work too late, resulting in underutilized resources and late delivery to customers. Overestimating lead times, on the other hand, will cause high WIP levels with the associated inventory costs as well as limiting the firm's ability to react to demand changes in a timely manner. Long lead times for production systems may also require higher safety stock levels in the supply chain, resulting in additional costs.

In this paper we address an aspect of this problem in the specific context of LP models for production planning. We consider a finite time horizon divided into discrete periods of equal length. The objective of the model is to determine the amount of each product to release into the production system in each period so as to maximize the profit achieved by the system. Multiple resource types with limited capacity are considered. Two different types of lead time estimates are considered: a conventional treatment requiring lead times to be an integer multiple of the underlying planning period, and an enhanced formulation using the approach of Hackman and Leachman (1989) to incorporate non-integer lead times. We evaluate the performance of the two models under a range of operating conditions by simulating the execution of the release schedules determined by the models.

In the following section we give a brief review of previous related work. Section 3 presents the LP models used, and Section 4 the wafer fabrication system used as a testbed as well as the simulation environment used to implement our experiments. Results are presented in Section 5, and a discussion of conclusions and directions for future research concludes the paper.

\section{PREVIOUS RELATED WORK}

Production planning has been addressed using the tools of operations research since the early work of Holt et al. (1960), Modigliani and Hohn (1955) and Manne (1957), among others. The use of LP models for these problems assumes a finite planning horizon divided into discrete time periods. Decision variables are associated with each time period, usually representing the amount of work released into each resource in each period. These decision variables can then be used to compute the values of a number of state variables, such as work in process and finished goods inventory levels, which can be used to estimate the costs incurred by the production plan. Two types of constraints are required for each planning period. The first set ensures material balance, i.e., the conservation of material flow, across periods. The second group enforces aggregate capacity constraints on the maximum amount of work that can be processed at each resource, or set of resources, in a period. Detailed discussions of these models are given by 
Johnson and Montgomery (1974), Missbauer and Uzsoy (2010), and Voss and Woodruff (2003), among others. Two basic approaches to the treatment of lead times dominate the literature on optimization models for production planning. The first of these, the most prevalent, treats lead times as an exogenous, workload-independent parameter to be estimated. These models generally assume that the lead time is an integer multiple of the underlying planning period. However, Hackman and Leachman (1989) show that the extension to non-integer lead times is straightforward under the assumption that activity levels such as production rates and material flows remain constant over each planning period. The second approach consists of multi-model approaches combining linear programming and simulation in an iterative scheme. A typical approach is that of Hung and Leachman (1996), which uses an LP model with non-integer lead times. Initial estimates of lead times are used to develop an initial release plan. The execution of this release schedule is then simulated using a detailed model of the production facility being planned, and the realized cycle times observed. These realized cycle times are then used to develop updated lead time estimates which are used in the LP model to develop a revised release schedule. The process continues until convergence is achieved according to some criterion. A range of models of this type have been proposed in the literature (Byrne and Bakir 1999; Hung and Hou 2001; Kim and Kim 2001; Byrne and Hossain 2005; Bang and Kim 2010; Irdem et al. 2010; Kacar et al. 2012). These approaches are outside the scope of this study, but depend on the use of LP formulations of the type discussed in this paper.

\section{LINEAR PROGRAMMING MODELS}

In this section we present the LP models that will be compared in the simulation experiments. We will first define the common notation, and then present the two LP models. The linear programming models we use are based on the Step-Separated formulation of Leachman and Carmon (1992), with the difference that our models do not allow alternative resources for operations, i.e., each operation in a product routing has exactly one predecessor and one successor, except for the first and last operations in the routing. This formulation assumes that the primary objective of the planning model is to determine the quantity of each product to be released into the fab in each period, rather than specific production quantities of each operation. Hence material that is processed at one operation is assumed to become available to the next operation on the product's routing immediately upon the completion of its processing at the current operation, and strategic inventory is not held anywhere in the line except after the final operation. We shall first present the formulation assuming integer lead times. We then highlight the deficiencies of this formulation in the presence of non-integer lead times, and present the modified formulation of Hackman and Leachman (1989) that addresses these issues.

\subsection{Notation}

We define an operation $l$ to be the processing of a product $g$ at a specific workcenter $k$ which forms the basis for the following notation:

\section{Sets:}

$O(g)$ : set of all operations of product $g$.

$O(k)$ : set of all operations performed on machines of workcenter $k$.

$T(g)$ : set of all time points internal to a planning period where the input rate to the finished inventory of product $g$ may change due to a non-integer lead time.

Indices:

$t$ : period index.

$g:$ product index.

$k$ : workcenter index.

$l$ : operation index. 
Decision Variables:

$Y_{g t l} \quad$ : quantity of product $g$ completing the $l$ 'th operation in period $t$.

$Y_{g t} \quad$ : output quantity of product $g$ in period $t$ from the last operation in its routing.

$X_{g t} \quad$ : quantity of product $g$ released into the first station in the line in period $t$.

$W_{g t} \quad$ : WIP of product $g$ at the end of period $t$.

$I_{g t} \quad$ : units of product $g$ in finished goods inventory at the end of period $t$.

$B_{g t} \quad$ : units of product $g$ backlogged at the end of period $t$.

Parameters:

$h_{g t} \quad$ : unit inventory holding cost for product $g$ in period $t$.

$b_{g t} \quad$ : unit backlogging cost for product $g$ in period $t$.

$\omega_{g t} \quad$ : unit WIP cost for product $g$ in period $t$.

$D_{g t} \quad$ : demand for product $g$ during period $t$.

$C_{k} \quad$ : maximum capacity of workcenter $k$ in units of products.

$\alpha_{g l} \quad$ : processing time of operation $l$ of product $g$.

$F F g$ : estimated flow factor of product $g$.

$L(g, l)$ : estimated time elapsing from the release of the raw material of product $g$ to the completion of the $l$ 'th operation of product $g$.

\subsection{Integer Lead Time Model}

The formulation, which is similar to that of Leachman and Carmon (1992) as follows:

Objective function:

$\min \sum_{g \in G} \sum_{t=1}^{T}\left[\omega_{g t} W_{g t}+h_{g t} I_{g t}+b_{g t} B_{g t}\right]$

Subject to:

WIP Balance:

$W_{g t}=W_{g, t-1}+X_{g t}-Y_{g t}$, for all $g \in G, t=1, \ldots, T$

Finished Inventory Balance:

$Y_{g t}+I_{g, t-1}-I_{g t}+B_{g t}-B_{g, t-1}=D_{g t}$, for all $t=1, \ldots, T, g \in G$

Fixed Lead Time Definition:

$Y_{g t l}=X_{g, t-L(g, l)}$, for all $t=1, \ldots, T, l \in O(g), g \in G$

Capacity Constraints:

$\sum_{l \in O(k)} \alpha_{l} Y_{g t l} \leq C_{k}$, for all $t=1, \ldots, T, k=1, \ldots, K$

Variable Nonnegativity:

$W_{g t}, Y_{g t l}, X_{g t}, I_{g t}, B_{g t} \geq 0$ for all $t=1, \ldots, T, g \in G, l \in O(g)$ 
We include WIP variables and constraints (2) in contrast to the conventional fixed lead time LPs proposed in the literature in order to compute the WIP cost in the objective function. Constraint set (3) represents material balance at the inventory of finished products at the end of the production line. Constraints (4) define the relation between the time a lot of product $g$ is released into the fab and its completing processing at operation $l$ of product $g$. This constraint is included for clarity of exposition; in practice an appropriate substitution would be used to eliminate the $Y_{g t l}$ variables. As soon as a lot is processed at a given operation, it becomes available to the next operation on its routing. Constraint (5) ensures that the total time required to process all operations processed at each workcenter in a given period $t$ does not exceed the time available at that workcenter; note that the model assumes capacity is consumed by an operation in the period that the operation is processed. We now discuss the issues that arise when non-integer lead times are present, and the modifications to the formulation required to address these.

\subsection{Non-integer Lead Time Model}

The reader will note that in the formulation above, the relationship between the releases $X_{g t}$ of a product $g$ in period $t$ and the amount of capacity consumed by a given operation at some future period is of the nature of a simple time delay. All $X_{g t}$ units of material of product $g$ released into the fab in period $t$ will consume capacity at operation $l \in O(g)$ in period $t+L(g, l)$. Thus all material processed at a particular operation in a period $t$ will correspond to the amount of the product concerned that is released in a single period. However, when non-integer lead times are involved, the amount of material $Y_{g t l}$ processed at an operation in period $t$ can be made up of material released during more than one period. Figure 1illustrates the basic issue involved, using a generic operation $j$ of product 1 with $L(1, j)=1.5$ periods. The amount of product released in each period $t$ is denoted on the upper timeline by $X_{1 t}$, and the amount of material to be processed at the operation $j$ in period $t$ as $Y_{l j t}$ on the lower timeline. Examining period 5 for purposes of illustration, it is clear that the material processed at the operation in period 5 will be composed of some material released in the latter part of period 3, and some from the first part of period 4. Assuming that releases in a planning period are uniformly distributed over the planning period, we see that

$Y_{g t l}=\varphi_{g l} X_{g, t-\lceil L(g, l)\rceil}+\left(1-\varphi_{g l}\right) X_{g, t-\lfloor L(g, l)\rfloor}$

where $\varphi_{g l}=L(g, l)-\lfloor L(g, l)\rfloor$ represents the fractional portion of the lead time $L(g, l)$. We note that this is a special case of the more general treatments of non-integer lead times given by Hackman and Leachman (Hackman and Leachman 1989) and Hackman (2008), who consider a continuous time model as opposed to the discrete time model considered here, but assume, as we do, that lead times remain constant over the planning horizon. Hung and Leachman (1996) consider the more general situation where lead times may vary over time, and propose a slightly different approach based on associating lead times with the beginning and ending points of planning periods which we do not consider here.

The use of non-integer lead times raises another issue in the context of the material balance constraints (3). LP models assume that all activity rates such as production, releases or demand are constant during each planning period. Hence the rate of inflow (production) into and outflow (demand) from any inventory location cannot change except at the boundaries of periods, and it is sufficient to enforce material balance at these points alone, as in constraints (3) above. However, when non-integer lead times are present, input rates into an inventory may change during a planning period, as is the case in period 5 shown in Figure 1. Hackman and Leachman (1989) point out two possible solutions to this issue. 
Kacar, Mönch, and Uzsoy

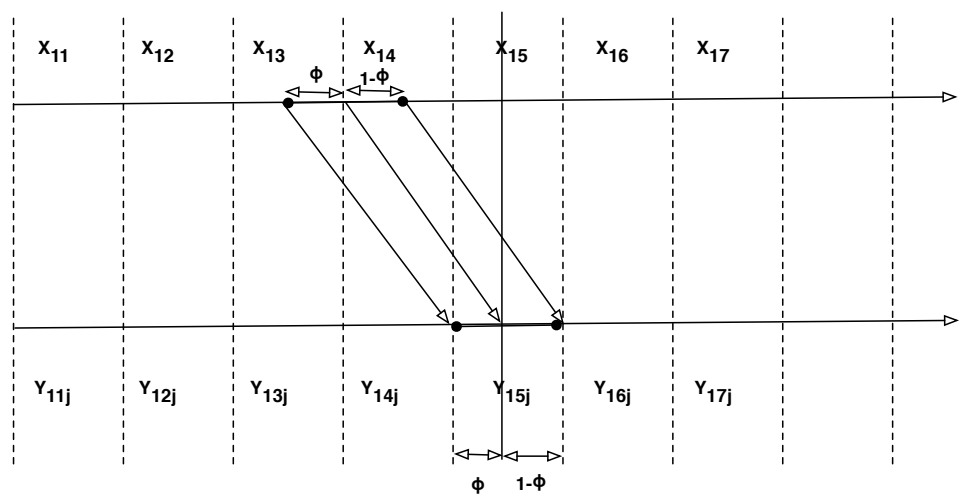

Figure 1: Accounting for output or loading in a period with non-integer lead times

The first is to reduce the length of each planning period to obtain integer lead times, or by writing additional material balance constraints at the points in time where input and output rates into the inventory location may change. These points can easily be identified based on the observation, implicit in the relationship (7) above, that for any point in time $t$ where a rate will change, $t-L(g, l)$ must be an integer. We will denote the set of time points at which such constraints must be written for product $g$ as $T(g)$, and compute WIP and finished inventory levels as well as backlogs at all these points. Based on these insights, the formulation for non-integer lead times can now be given as follows:

Objective function:

$\min \sum_{g \in G} \sum_{t \in T(g)}\left[\omega_{g t} W_{g t}+h_{g t} I_{g t}+b_{g t} B_{g t}\right]$

Subject to:

WIP Balance:

$W_{g t}=W_{g, t-1}+X_{g t}-Y_{g t}$, for all $g \in G, t=1, \ldots, T$

Inventory Balance:

$Y_{g t}+I_{g, t-1}-B_{g, t-1}-I_{g t}+B_{g t}=D_{g t}$, for all $t=1, \ldots, T, g \in G$

Input Rate Change Epoch Constraints:

$\varphi_{g l} X_{g, t-\lceil L(g, l)\rceil}+I_{g, t-1}-I_{g \tau}-B_{g, t-1}+B_{g \tau}=\varphi_{g l} D_{g t}$, for $t=1, \ldots, T, \tau \in T(g), g \in G, l \in O(g)$

Non-integer Lead Time Constraints:

$Y_{g t l}=\varphi_{g l} X_{g, t-\lceil L(g, l\rceil}+\left(1-\varphi_{g l}\right) X_{g, t-\lfloor L(g, l)\rfloor}$, for all $t=1, \ldots, T, g \in G, l \in O(g)$

Capacity Constraints:

$\sum_{g \in G, k \in K} \alpha_{g l} Y_{g t l} \leq C_{k}$, for all $t=1, \ldots, T, k \in K$

Variable Nonnegativity:

$W_{g t}, X_{g t}, Y_{g t l}, I_{g t}, B_{g t} \geq 0$ for all $t=1, \ldots, T, g \in G, l \in O(g)$ 
The constraint set (11) captures the inventory position at the points of rate change within the period. It only includes the last operation of each product since the interest is on finished goods instead of intermediate inventories of operations in the factory. Those inventory positions at the points of rate change are included in the objective function and same cost values of inventory and backlog are used. The constraint (5) represents the capacity loading of machines in a period loaded by releases from several periods due to the non-integer lead time. Note that the lead time estimates are constant across the periods; therefore the constraints consist of two parts which sum the fraction of releases with the consideration of the rounded lead time values.

\section{SIMULATION ENVIRONMENT}

\subsection{Simulation Model}

We consider the full MIMAC I model (Fowler and Robinson 1995) which is publicly available from the University of Hanover. It contains over 200 machines organized into 84 workcenters. Workcenters with batching machines and sequence-dependent setup times are included. The model contains two process flows with 210 and 245 process steps, respectively. The product flows are highly re-entrant. All processing times are deterministic, and depend either on the number of wafers in a lot or are determined for each lot. The model assumes instantaneous material transfer between consecutive process steps on a given route. Operators are not included in our model, and rework is not considered. The First-In First-Out (FIFO) rule is used to dispatch lots in front of the workcenters. A minimum number of lots is required to form batches at each batch processing workcenter to mimic minimum batch size policies. Only lots at the same processing step (operation) can be batched together.

We adjusted the number of steppers to ensure that this workcenter serves as a planned bottleneck if a product mix of 1:1 is assumed. Lots to be released in a given week are distributed uniformly over this time period. Each lot contains 48 wafers. The variability in the system is caused by exponentially distributed machine breakdowns. Although there is some evidence from the literature that Weibull distributions are more suitable for modeling breakdowns in wafer fabs, we decided to use the original breakdown data from the reference model. In order to extract the non-integer lead times for each operation $l$, we use the following equation:

$$
L(g, l)=\alpha_{g l} F F_{g}+L(g, l-1), l \in O(g), g \in G .
$$

Recall that $\alpha_{g l}$ is the processing time of product $g$ at operation $l$ in minutes and $O(g)$ the set of operations that the product goes through in order. The lead time for each operation is calculated from the time that the product is released into the plant, yielding the cumulative calculation in (15). $F F_{g}$ denotes the flow factor for product $g$, defined as the ratio of the average flow time of the product through the fab to the sum of all operation processing times on its routing. As the lead time of each operation depends on that of the previous operation in its routing this expression is recursive in nature. For the first operation of each product $g$ it reduces to $L(g, 1)=\alpha_{g 1} F F_{g} \alpha_{g l}$.

\subsection{Design of Experiments}

We expect the performance of the production planning models to depend on the level of resource utilization and the degree of processing variability at each workcenter. Therefore we generate normally distributed demand for each product in each period to obtain mean bottleneck utilization levels of $70 \%$ and $90 \%$. The utilization levels are achieved by varying the demand of the two products while maintaining the same mean demand for both products. We consider a planning horizon of 15 one-week periods where weekly releases are to be determined for each product. We also study the effect of demand variability by consid- 
ering values of 0.1 and 0.25 for the coefficient of variation (CV) of demand for each product. Five independent demand realizations are generated for each combination of utilization and variability levels, which we shall refer to as a demand scenario. Thus we consider four demand scenarios.

We determine an initial WIP distribution of the lots to reduce the initialization effects for each demand realization of a given demand scenario. Five independent simulation runs are conducted for one year (52 weeks), and a snapshot of the location of lots in the system after 37 weeks is taken. One of these five different initial WIP distributions is selected randomly for each simulation replication. The demand for the remaining 15 weeks is used as the demand in this simulation experiment.

To examine the effects of variability, we consider long and short failure durations. We take the exponentially distributed Mean Time to Failure (MTTF) and Mean Time to Repair (MTTR) values in the original MIMAC model to represent the short failure case. The long failure case is obtained by doubling the MTTR and the MTTF values yielding the same average availability as in the short failure scenario.

Simulation of the wafer fab serving as the testbed in this study revealed that the cycle times for most operations were less than one planning period, and the average cycle time for the overall line was of the order of three periods. Hence the integer lead time model requires a method of obtaining integer lead times from the non-integer values observed in the simulation. This was achieved using two different approaches. The unit revenue value is 180 , while the unit WIP, backlog, and inventory costs are 35,50 , and 15 , respectively.

\subsubsection{Simple Rounding Down (SRD)}

In the SRD scheme we obtain integer lead times by rounding these estimates $L t(g, l)$ down. The SRD procedure assumes that as long as product $g$ is released $L t(g, l)$ periods before it reaches its $l$ 'th operation, the processing of that operation $l$ will be completed within that period. Suppose that the lead time $L t(g, l)$ of product $g$ at operation $l$ is 2.5 periods. The SRD procedure will round it down to 2 periods, implying that the output of operation $l$ is determined by releases into the plant 2 periods earlier. The obvious disadvantage of this procedure is that we may severely underestimate the lead times of some operations. In order to address this problem, we introduce an integer programming model where some lead times are rounded down and some are rounded up.

\subsubsection{Integer Programming Rounding (IPR)}

Consider an observed lead time of 2.9 periods. The SRD procedure will round it down to 2 periods, yielding an error of 0.9 periods. On the other hand, if we were to round it up to 3 periods the error is reduced to 0.1 periods which is more reasonable. The following integer program suggested by Turkseven (2005) rounds some lead times up and others down instead of simply rounding all values down. We define the

decision variables $\bar{L}(g, l)$ to be the integer $\bar{L}$ lead time of product $g$ at operation $l$. Recall that $L(g, l)$ is the non-integer lead time estimate calculated using the flow factor estimate explained in the previous approach. The integer program is as follows:

$\min \sum_{g \in G, l \in O(g)} \bar{L}(g, l)$

Subject to:

$$
\begin{aligned}
& \sum_{l \in O(g)} L(g, l) \leq \sum_{l \in O(g)} \bar{L}(g, l), g \in G \\
& \bar{L}(g, l)-L(g, l) \leq 1, g \in G, l \in O(g) \\
& L(g, l)-\bar{L}(g, l) \leq 1, g \in G, l \in O(g) \\
& \bar{L}(g, l-1) \leq \bar{L}(g, l), g \in G, l \in O(g) \\
& \bar{L}_{(g, l)} \in Z^{+}
\end{aligned}
$$


The objective function (16) minimizes the sum of the new integer lead time estimates $\bar{L}$ subject to the constraint (17) that their cumulative sum has to be at least equal to the value of the cumulative sum of the non-integer lead times. The constraints (18) and (19) ensure that lead times will be either rounded up or down. The constraint (20) ensures that the lead time estimate of an operation has to be at least equal to that of the previous operation on its routing. Recall that lead times are calculated from the time of the release of material into the plant. Lead time estimates are integer values as shown in (21). Constraint (17) will ensure that we do not simply round down the values. After we obtain our lead time estimates $\bar{L}(g, l)$ from the IP model above, we will set $L(g, l)=\bar{L}(g, l)$ in the fixed lead time LP model presented above.

In our computational experiments we examine how these different approaches to estimating integer lead times affect the performance of the LP-FL model. We solve each of the three planning models for each demand replication and perform 20 independent simulation runs to estimate the realized performance measures. The design of experiments is summarized in Table 1.

Table 1: Summary of Experimental Design

\begin{tabular}{|c|c|c|}
\hline Factor & Level & Count \\
\hline Mean demand & High, Low & 2 \\
\hline CV of demand & High, Low & 2 \\
\hline Failure duration & Long, Short & 2 \\
\hline Planning Models & SDR, IPR, FLP & 3 \\
\hline $\begin{array}{c}\text { Replications per demand sce- } \\
\text { nario }\end{array}$ & & 5 \\
\hline $\begin{array}{c}\text { Simulation replications per } \\
\text { demand realization }\end{array}$ & & 20 \\
\hline Total simulation runs & & 2400 \\
\hline
\end{tabular}

\section{RESULTS OF EXPERIMENTS}

The results of the experiment are summarized in Figure 2. It is apparent that the use of non-integer lead times significantly enhances the performance of the production plans obtained from the LPs across all experimental conditions. The consistently superior performance of the FLP model suggest that it is better able to represent the dynamics of capacity consumption over time in this complex reentrant production system that the SRD and IPR models with integer lead times. In Table 2 we compare the ratio of the average realized profit from the SRD and IPR models to those from the FLP model for each experimental condition. We obtain increases in expected profit of up to $30 \%$ in some cases, suggesting that the use of the non-integer lead times yields significant benefits over the use of integer lead times.

Examination of the cost distributions on the right hand side of Figure 2 provides insight into the causes of the differences in performance. The SRD model consistently incurs high backorder costs and holds almost no finished inventory. This implies that it systematically underestimates the lead times, causing material to be released too late to meet demand in a timely manner. Given the nature of the rounding procedure used in SRD, this is not surprising. The performance of the IPR model is more varied. In general, it incurs lower backlogs than SRD, and higher finished inventory costs. When compared to the FLP model, IPR generally yields both higher backlogs and higher finished inventory costs, suggesting that its ability to match output to demand over time is not as good as that of the FLP model. This is probably due to differences in the lead times for individual operations, some of which will be rounded up and others rounded down by the IPR procedure. The lead times for individual operations do not affect the flow of material into the finished inventory at the end of the line, but do determine the way in which the capacity constraints at the machines will affect the planned releases. 
In order to assess the statistical significance of the results, we compared the results of the different planning models for each demand realization using the Friedman test (Conover 1980). While we do not present details of this analysis for the sake of brevity, in all experimental conditions the FLP model outperformed the SRD and IPR models at a significance level of 0.95 .
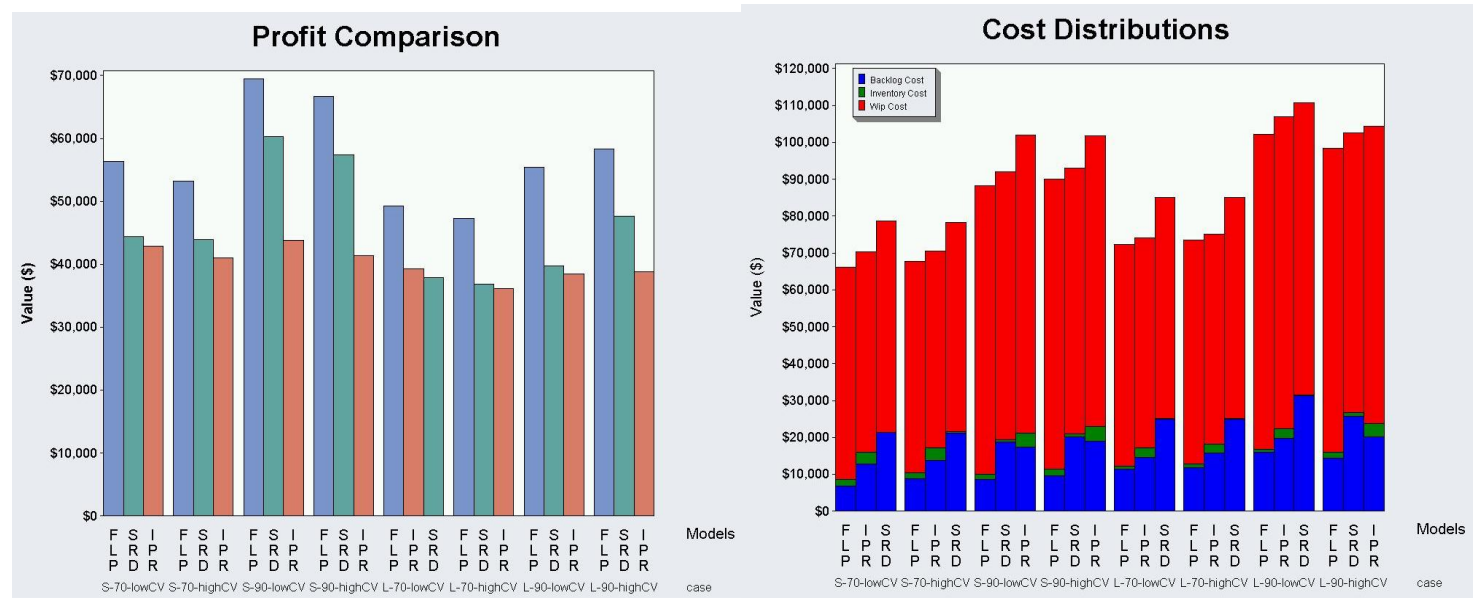

Figure 2: Comparison of Average Realized Profit and Cost Components from Planning Models

Table 2: Ratios of Expected Realized Profit Relative to FLP Model

\begin{tabular}{|c|c|c|c|c|c|c|c|c|}
\hline & S-70-LowCV & S-70-HighCV & S-90-LowCV & S-90-HighCV & L-70-LowCV & L-70-HighCV & L-90-LowCV & L-90-HighCV \\
\hline IPR/FLP & 0.76 & 0.77 & 0.63 & 0.62 & 0.80 & 0.76 & 0.69 & 0.67 \\
SRD/FLP & 0.79 & 0.83 & 0.87 & 0.86 & 0.77 & 0.78 & 0.72 & 0.82 \\
\hline
\end{tabular}

\section{CONCLUSIONS AND FUTURE DIRECTIONS}

The experiments presented in this paper demonstrate that under the right conditions the incorporation of non-integer lead times can significantly enhance the performance of linear programming models for production planning. The FLP model yields significant improvements in expected realized profit over both of the alternative models using integer lead times. The use of non-integer lead times requires additional constraints and variables, but the larger formulation is still computationally tractable.

A number of limitations of our study need to be noted at this point. First of all, the experimental environment we have implemented is very favorable to the use of non-integer lead times. The demand distribution faced by the production facility is stationary over time, allowing accurate estimates of lead times for each workcenter based on historic data (in our case, obtained from a long simulation run prior to the start of the planning problem). Under these conditions, the average utilization of the resources can be expected to be quite stable from period to period, allowing time-stationary lead time estimates to be quite accurate. In the event of demand, and hence resource utilization, varying significantly from one time period to the next, the question of how to estimate the non-integer lead times begins to be challenging. An obvious approach is to use a simulation model for this purpose prior to running the planning models, but this requires knowledge of the release schedules that will be developed by the planning model after it is run. The incorporation of the FLP model into the iterative simulation-LP approach of Hung and Leachman (1996) would seem to offer another avenue of attack, which must be examined in future work. Final- 


\section{Kacar, Mönch, and Uzsoy}

ly, a comparison of the FLP model with the clearing function approaches that have proven superior to LP models with integer lead times is another interesting direction for future work.

\section{ACKNOWLEDGMENTS}

The research of Reha Uzsoy was supported by the National Science Foundation under Grant No. CMMI1029706.

\section{REFERENCES}

Asmundsson, J. M., R. L. Rardin, C. H. Turkseven, and R. Uzsoy (2009). "Production Planning Models with Resources Subject to Congestion." Naval Research Logistics 56: 142-157.

Asmundsson, J. M., R. L. Rardin, and R. Uzsoy (2006). "Tractable Nonlinear Production Planning Models for Semiconductor Wafer Fabrication Facilities." IEEE Transactions on Semiconductor Manufacturing 19: 95-111.

Baker, K. R. (1993). Requirements Planning. Handbooks in Operations Research and Management Science. S. C. Graves, A. H. G. Rinnooy Kan and P. H. Zipkin. Amsterdam, Elsevier Scence Publishers. Volume 3: Logistics of Production and Inventory: 571-627.

Bang, J. Y., and Y. D. Kim (2010). "Hierarchical Production Planning for Semiconductor Wafer Fabrication Based on Linear Programming and Discrete-Event Simulation." IEEE Transactions on Automation Science and Engineering 7(2): 326-336.

Buzacott, J. A., and J. G. Shanthikumar (1993). Stochastic Models of Manufacturing Systems. Englewood Cliffs, NJ, Prentice-Hall.

Byrne, M. D., and M. A. Bakir (1999). "Production Planning Using a Hybrid Simulation-Analytical Approach." International Journal of Production Economics 59: 305-311.

Byrne, M. D. and M. M. Hossain (2005). "Production Planning: An Improved Hybrid Approach." International Journal of Production Economics 93-94: 225-229.

Conover, W. J. (1980). Practical Nonparametric Statistics. New York, John Wiley.

Fowler, J. and J. Robinson (1995). Measurement and Improvement of Manufacturing Capacities (MIMAC): Final Report. . Technical report 95062861A-TR. Austin, TX, SEMATECH.

Hackman, S. (2008). Production Economics. Berlin, Springer.

Hackman, S. T., and R. C. Leachman (1989). "A General Framework for Modeling Production." Management Science 35: 478-495.

Holt, C. C., F. Modigliani, J. F. Muth, and H. A. Simon (1960). Planning Production, Inventories and Work Force. Englewood Cliffs, NJ, Prentice Hall.

Hopp, W. J., and M. L. Spearman (2008). Factory Physics : Foundations of Manufacturing Management. Boston, Irwin/McGraw-Hill.

Hung, Y. F., and M. C. Hou (2001). "A Production Planning Approach based on Iterations of Linear Programming Optimization and Flow Time Prediction." Journal of the Chinese Institute of Industrial Engineers 18(3): 55-67.

Hung, Y. F., and R. C. Leachman (1996). "A Production Planning Methodology for Semiconductor Manufacturing Based on Iterative Simulation and Linear Programming Calculations." IEEE Transactions on Semiconductor Manufacturing 9(2): 257-269.

Irdem, D. F., N. B. Kacar, and R. Uzsoy (2010). "An Exploratory Analysis of Two Iterative Linear Programming-Simulation Approaches for Production Planning." IEEE Transactions on Semiconductor Manufacturing 23: 442-455. 
Johnson, L. A., and D. C. Montgomery (1974). Operations Research in Production Planning, Scheduling and Inventory Control. New York, John Wiley.

Kacar, N. B., D. F. Irdem, and R. Uzsoy (2012). "An Experimental Comparison of Production Planning using Clearing Functions and Iterative Linear Programming-Simulation Algorithms." IEEE Transactions on Semiconductor Manufacturing 25(1): 104-117.

Kim, B., and S. Kim (2001). "Extended Model for a Hybrid Production Planning Approach." International Journal of Production Economics 73: 165-173.

Leachman, R. C., and T. F. Carmon (1992). "On Capacity Modeling for Production Planning with Alternative Machine Types." IIE Transactions 24(4): 62-72.

Manne, A. S. (1957). "A Note on the Modigliani-Hohn Production Smoothing Model." Management Science 3(4): 371-379.

Missbauer, H., and R. Uzsoy (2010). Optimization Models for Production Planning. Planning Production and Inventories in the Extended Enterprise: A State of the Art Handbook. K. G. Kempf, P. Keskinocak and R. Uzsoy. New York, Springer: 437-508.

Modigliani, F., and F. E. Hohn (1955). "Production Planning Over Time and the Nature of the Expectation and Planning Horizon." Econometrica 23(1): 46-66.

Orlicky, J. (1975). Material Requirements Planning: the New Way of Life in Production and Inventory Management. New York, McGraw-Hill.

Pahl, J., S. Voss, and D. L. Woodruff (2005). "Production Planning with Load Dependent Lead Times." 4OR: A Quarterly Journal of Operations Research 3: 257-302.

Pahl, J., S. Voss, and D. L. Woodruff (2007). "Production Planning with Load Dependent Lead Times: An Update of Research." Annals of Operations Research 153: 297-345.

Turkseven, C. H. (2005). Computational Evaluation of Production Planning Formulations Using Clearing Functions. School of Industrial Engineering. West Lafayette, IN, Purdue University.

Voss, S., and D. L. Woodruff (2003). Introduction to Computational Optimization Models for Production Planning in a Supply Chain. Berlin ; New York, Springer.

\section{AUTHOR BIOGRAPHIES}

NECIP BARIS KACAR is an Operations Research Specialist at the SAS Institute. He holds a Ph.D. degree in Industrial Engineering with Minor in Operations Research from the Edward P. Fitts Department of Industrial and Systems Engineering at North Carolina State University, and also holds a M.S. from the same university. He received a BS degree in Mechanical Engineering from Bogazici University, Istanbul, Turkey. His research interests are in production planning, supply chain management, inventory optimization and simulation based optimization. He can be reached via email at $<$ Baris.Kacar@sas.com>.

LARS MÖNCH is full professor of Computer Science at the Department of Mathematics and Computer Science, University of Hagen where he heads the Chair of Enterprise-wide Software Systems. He holds MS and Ph.D. degrees in Mathematics from the University of Göttingen, Germany. After his Ph.D., he obtained a habilitation degree in Information Systems from Technical University of Ilmenau, Germany. His research and teaching interests are in information systems for production and logistics, simulation, scheduling, and production planning, He can be reached by email at $<$ lars.moench@fernuni-hagen.de>.

REHA UZSOY is Clifton A. Anderson Distinguished Professor in the Edward P. Fitts Department of Industrial and Systems Engineering at North Carolina State University. He holds BS degrees in Industrial Engineering and Mathematics and an MS in Industrial Engineering from Bogazici University, Istanbul, Turkey. He received his Ph.D. in Industrial and Systems Engineering in 1990 from the University of Florida. His teaching and research interests are in production planning, scheduling, and supply chain man- 
agement. He was named a Fellow of the Institute of Industrial Engineers in 2005, Outstanding Young Industrial Engineer in Education in 1997, and has received awards for both undergraduate and graduate teaching. He can be reached by email at $<$ ruzsoy@ncsu.edu>.

Asmundsson, J. M., R. L. Rardin, C. H. Turkseven and R. Uzsoy (2009). "Production Planning Models with Resources Subject to Congestion." Naval Research Logistics 56: 142-157.

Asmundsson, J. M., R. L. Rardin and R. Uzsoy (2006). "Tractable Nonlinear Production Planning Models for Semiconductor Wafer Fabrication Facilities." IEEE Transactions on Semiconductor Manufacturing 19: 95111.

Baker, K. R. (1993). Requirements Planning. Handbooks in Operations Research and Management Science. S. C. Graves, A. H. G. Rinnooy Kan and P. H. Zipkin. Amsterdam, Elsevier Scence Publishers. Volume 3: Logistics of Production and Inventory: 571-627.

Bang, J. Y. and Y. D. Kim (2010). "Hierarchical Production Planning for Semiconductor Wafer Fabrication Based on Linear Programming and Discrete-Event Simulation." IEEE Transactions on Automation Science and Engineering 7(2): 326-336.

Buzacott, J. A. and J. G. Shanthikumar (1993). Stochastic Models of Manufacturing Systems. Englewood Cliffs, NJ, Prentice-Hall.

Byrne, M. D. and M. A. Bakir (1999). "Production Planning Using a Hybrid Simulation-Analytical Approach." International Journal of Production Economics 59: 305-311.

Byrne, M. D. and M. M. Hossain (2005). "Production Planning: An Improved Hybrid Approach." International Journal of Production Economics 93-94: 225-229.

Conover, W. J. (1980). Practical Nonparametric Statistics. New York, John Wiley.

Fowler, J. and J. Robinson (1995). Measurement and Improvement of Manufacturing Capacities (MIMAC): Final Report Technical report 95062861A-TR. Austin, TX, SEMATECH.

Hackman, S. (2008). Production Economics. Berlin, Springer.

Hackman, S. T. and R. C. Leachman (1989). "A General Framework for Modeling Production." Management Science 35: 478-495.

Holt, C. C., F. Modigliani, J. F. Muth and H. A. Simon (1960). Planning Production, Inventories and Work Force. Englewood Cliffs, NJ, Prentice Hall.

Hopp, W. J. and M. L. Spearman (2008). Factory Physics : Foundations of Manufacturing Management. Boston, Irwin/McGraw-Hill.

Hung, Y. F. and M. C. Hou (2001). "A Production Planning Approach based on Iterations of Linear Programming Optimization and Flow Time Prediction." Journal of the Chinese Institute of Industrial Engineers 18(3): 55-67.

Hung, Y. F. and R. C. Leachman (1996). "A Production Planning Methodology for Semiconductor Manufacturing Based on Iterative Simulation and Linear Programming Calculations." IEEE Transactions on Semiconductor Manufacturing 9(2): 257-269.

Irdem, D. F., N. B. Kacar and R. Uzsoy (2010). "An Exploratory Analysis of Two Iterative Linear ProgrammingSimulation Approaches for Production Planning." IEEE Transactions on Semiconductor Manufacturing 23: $442-455$.

Johnson, L. A. and D. C. Montgomery (1974). Operations Research in Production Planning, Scheduling and Inventory Control. New York, John Wiley.

Kacar, N. B., D. F. Irdem and R. Uzsoy (2012). "An Experimental Comparison of Production Planning using Clearing Functions and Iterative Linear Programming-Simulation Algorithms." IEEE Transactions on Semiconductor Manufacturing 25(1): 104-117.

Kim, B. and S. Kim (2001). "Extended Model for a Hybrid Production Planning Approach." International Journal of Production Economics 73: 165-173.

Leachman, R. C. and T. F. Carmon (1992). "On Capacity Modeling for Production Planning with Alternative Machine Types." IIE Transactions 24(4): 62-72.

Manne, A. S. (1957). "A Note on the Modigliani-Hohn Production Smoothing Model." Management Science 3(4): 371-379.

Missbauer, H. and R. Uzsoy (2010). Optimization Models for Production Planning. Planning Production and Inventories in the Extended Enterprise: A State of the Art Handbook. K. G. Kempf, P. Keskinocak and R. 
Uzsoy. New York, Springer: 437-508.

Modigliani, F. and F. E. Hohn (1955). "Production Planning Over Time and the Nature of the Expectation and Planning Horizon." Econometrica 23(1): 46-66.

Orlicky, J. (1975). Material Requirements Planning: the New Way of Life in Production and Inventory Management. New York, McGraw-Hill.

Pahl, J., S. Voss and D. L. Woodruff (2005). "Production Planning with Load Dependent Lead Times." 4OR: A Quarterly Journal of Operations Research 3: 257-302.

Pahl, J., S. Voss and D. L. Woodruff (2007). "Production Planning with Load Dependent Lead Times: An Update of Research." Annals of Operations Research 153: 297-345.

Turkseven, C. H. (2005). Computational Evaluation of Production Planning Formulations Using Clearing Functions. School of Industrial Engineering. West Lafayette, IN, Purdue University.

Voss, S. and D. L. Woodruff (2003). Introduction to Computational Optimization Models for Production Planning in a Supply Chain. Berlin ; New York, Springer. 